\title{
Detection of the left accessory pathway with synthesized 18-lead electrocardiography in WPW syndrome
}

\section{Keiji Matsunaga, Takahisa Noma, Yuichi Miyake, Makoto Ishizawa, Teppei Tsuji and Tetsuo Minamino*}

Department of Cardiorenal and Cerebrovascular Medicine, Faculty of Medicine, Kagawa University, 1750-1, Ikenobe, Miki-cho, Kita-gun, Kagawa 761-0793, Japan

The synthesized 18-lead electrocardiography (18 lead-ECG) provides right-sided leads (V3R, V4R and V5R) and back leads (synV7, synV8 and synV9) via a mathematical computation so that only the standard 12 leads are used, and additional leads or techniques are not required [1]. 18 lead-ECG is useful for diagnosing the origin of a premature ventricular contraction and the risk area of myocardial infarction [2].

If we analyze the 18 lead-ECG, we can easily detect the delta wave polarity change because normal chest leads, and synthesized back leads are positioned to surround the mitral annulus (Figure 1A). Since the polarity of the delta wave is the same as the polarity of ventricular extrasystoles from the mitral annulus, we can determine the location of the left accessory pathway.

We show the 18 lead-ECG from a patient (Figure 1B) (recording system: ECG-2550: Nihon Kohden, Inc. viewer: ECG-Viewer Lite: Nihon Kohden, Inc.). The delta wave polarity was positive in V5, slightly

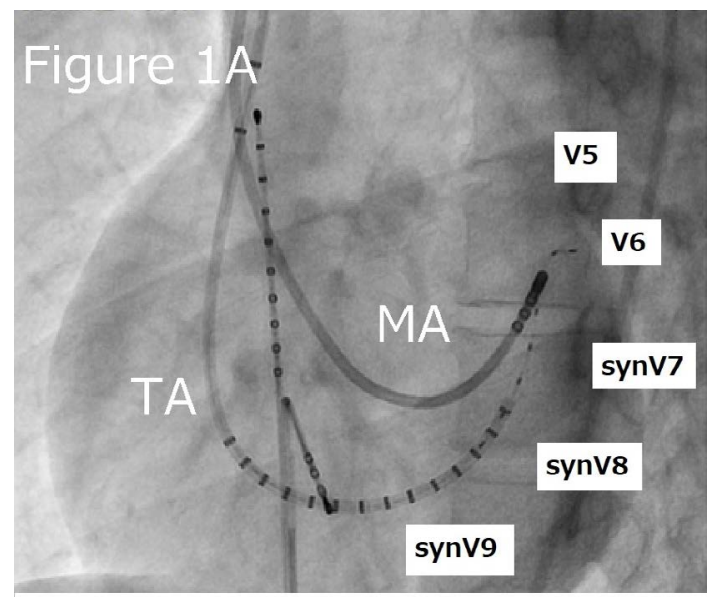

$\uparrow$ LAO $45^{\circ}$ TA: tricuspid annulus MA: mitral annulus

Figure 1A. Virtual placement of the leads and successful ablation point for the left accessory pathway

Copyright: (C2018 Matsunaga K. This is an open-access article distributed under the terms of the Creative Commons Attribution License, which permits unrestricted use, distribution, and reproduction in any medium, provided the original author and source are credited.

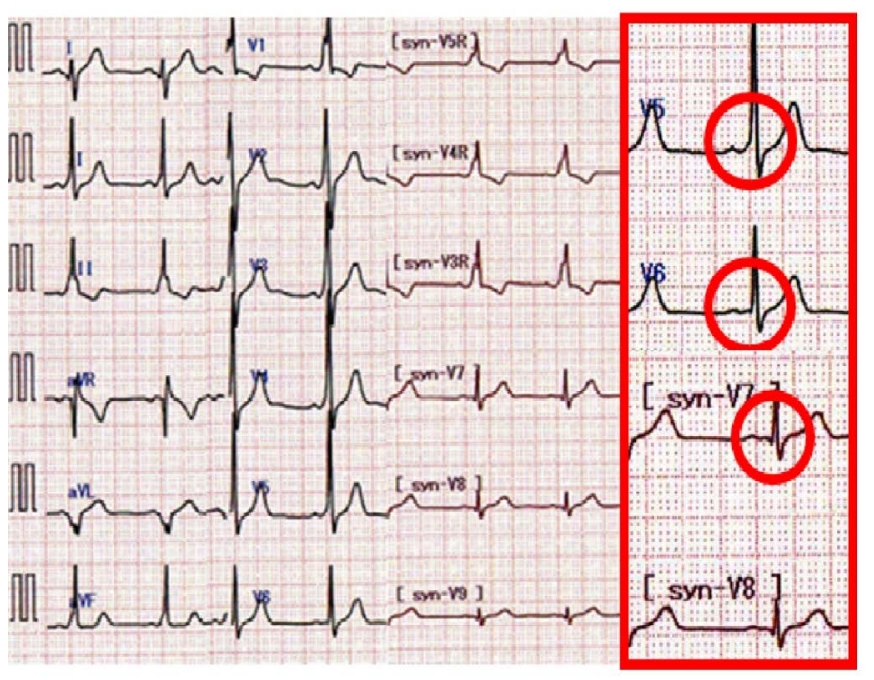

Figure 1B. Synthesized 18-lead electrocardiography results for a patient

positive in V6 and slightly negative in V7; thus, the delta wave polarity allowed us to speculate that the accessory pathway was located in the left lateral, and we can determine the location of the left accessory pathway before performing an electrophysiological study (EPS). The routine 18 lead-ECG before and after EPS will give us important information that will shorten the EPS time and confirm the successful ablation of the left accessory pathway.

\section{References}

1. Igarashi M, Nogami A, Sekiguchi Y, Kuroki K, Yamasaki H, et al. (2015) The QRS morphology pattern in V5R is a novel and simple parameter for differentiating the origin of idiopathic outflow tract ventricular arrhythmias. Europace 17: 1107-1116. [Crossref]

2. Ashida T, Tani S, Nagao K, Yagi T, Matsumoto N, et al. (2017) Usefulness of synthesized 18-lead electrocardiography in the diagnosis of ST-elevation myocardial infarction: A pilot study. Am J Emerg Med 35: 448-457. [Crossref]

${ }^{\star}$ Correspondence to: Tetsuo Minamino, Department of Cardiorenal and Cerebrovascular Medicine, Faculty of Medicine, Kagawa University, 1750-1, Ikenobe, Miki-cho, Kita-gun, Kagawa 761-0793, Japan, Tel: 81878912150, Fax: 8187891-2152, E-mail: minamino@med.kagawa-u.ac.jp

Key words: Wolff-Parkinson-White syndrome synthesized 18-lead electrocardiography, accessory pathway

Received: October 08, 2018; Accepted: October 24, 2018; Published: October 29,2018 